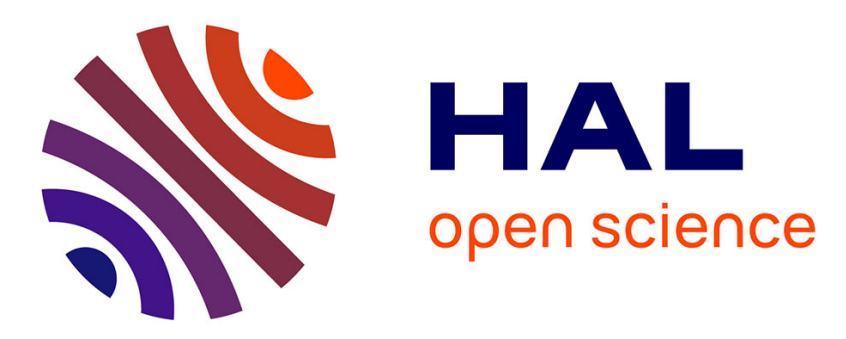

\title{
Pharmacokinetic/pharmacodynamic (PK/PD) considerations in the management of Gram-positive bacteraemia
}

Francesco Scaglione

\section{- To cite this version:}

Francesco Scaglione. Pharmacokinetic/pharmacodynamic (PK/PD) considerations in the management of Gram-positive bacteraemia. International Journal of Antimicrobial Agents, 2010, 36, 10.1016/j.ijantimicag.2010.11.011 . hal-00650372

\section{HAL Id: hal-00650372 \\ https://hal.science/hal-00650372}

Submitted on 10 Dec 2011

HAL is a multi-disciplinary open access archive for the deposit and dissemination of scientific research documents, whether they are published or not. The documents may come from teaching and research institutions in France or abroad, or from public or private research centers.
L'archive ouverte pluridisciplinaire HAL, est destinée au dépôt et à la diffusion de documents scientifiques de niveau recherche, publiés ou non, émanant des établissements d'enseignement et de recherche français ou étrangers, des laboratoires publics ou privés. 


\section{Accepted Manuscript}

Title: Pharmacokinetic/pharmacodynamic (PK/PD) considerations in the management of Gram-positive bacteraemia

Author: Francesco Scaglione

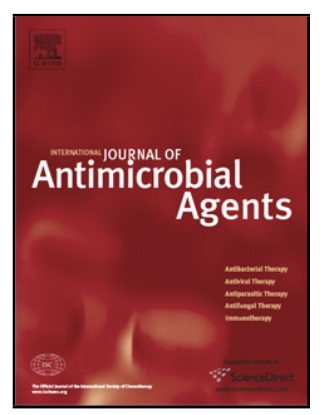

PII:

S0924-8579(10)00472-3

DOI: doi:10.1016/j.ijantimicag.2010.11.011

Reference: ANTAGE 3465

To appear in: International Journal of Antimicrobial Agents

Please cite this article as: Scaglione F, Pharmacokinetic/pharmacodynamic (PK/PD) considerations in the management of Gram-positive bacteraemia, International Journal of Antimicrobial Agents (2010), doi:10.1016/j.ijantimicag.2010.11.011

This is a PDF file of an unedited manuscript that has been accepted for publication. As a service to our customers we are providing this early version of the manuscript. The manuscript will undergo copyediting, typesetting, and review of the resulting proof before it is published in its final form. Please note that during the production process errors may be discovered which could affect the content, and all legal disclaimers that apply to the journal pertain. 
Pharmacokinetic/pharmacodynamic (PK/PD) considerations in the management of Gram-positive bacteraemia

Francesco Scaglione*

Department of Pharmacology, Chemotherapy and Toxicology, Faculty of Medicine, University of Milan, Via Vanvitelli 32, 20129 Milan, Italy

* Tel.: +39025031 7073; fax: +390250317050.

E-mail address: francesco.scaglione@unimi.it. 


\section{Abstract}

Bloodstream infections are among the most serious infections of hospitalized patients and are associated with high mortality, especially among those with severe sepsis and septic shock. A range of organ dysfunctions, together with drug interactions and other therapeutic interventions (e.g. haemodynamically active drugs and continuous renal replacement therapies) may have a strong impact on antimicrobial drug pharmacokinetics in critically ill patients. Intrinsic pharmacokinetic (PK) and pharmacodynamic (PD) properties are the major determinants of the in vivo efficacy of antimicrobial agents. Knowledge of PK/PD parameters is essential in facilitating the translation of microbiological activity into clinical situations and ensuring a successful outcome. This review analyses the typical patterns of antimicrobial activity of classes of agent commonly utilized against Gram-positive pathogens in hospital settings, and their corresponding PK/PD parameters, focusing on the PK/PD dosing approach.

Keywords:

Bloodstream infections

Antimicrobial agents

Pharmacokinetics

Pharmacodynamics 


\section{Introduction}

Bloodstream infections are among the most serious infections of hospitalized patients and are associated with high mortality, especially among those with severe sepsis and septic shock [1-4]. Some studies have demonstrated an important relationship between hospital mortality and inadequate empirical antimicrobial treatment of bacteraemia (Fig. 1.). It is not surprising that appropriate therapy is related to lower mortality, but what is surprising is that appropriate therapy is related to mortality ranging from $30 \%$ to $50 \%$, depending from the study.

[Fig. 1. here]

What does the term 'appropriate therapy' mean? It is usually defined as either the presence of antimicrobial agents directed against a specific class of microorganisms, or the administration of drugs to which the microorganism responsible for the infection is susceptible [5]. These definitions, in the mind of many clinicians, represent only the minimum, an appropriate dosing regimen also including early administration and the correct drug dose or schedule of administration. Despite appropriate dosing regimens, failure of antimicrobial therapy may occur due to impaired immunological function or the inability of the antimicrobial to achieve adequate concentrations at the infection site due to alterations in its pharmacokinetics resulting from underlying pathophysiological conditions.

In the last decade it has become apparent that the intrinsic pharmacokinetic (PK) and pharmacodynamic (PD) properties of antimicrobial agents are the major determinants of their in vivo efficacy and knowledge of them enables the use of optimal dosing regimens and the determination of clinically relevant susceptibility breakpoints [6]. In this paper 
the use of PK/PD relationships is discussed, with a special focus on agents against Gram-positive organisms.

\section{Pharmacodynamic measures of antimicrobial effects}

Antimicrobial pharmacodynamics is the discipline that attempts to link measures of drug exposure to their microbiological or clinical effects [7]. Only the free or unbound fraction of a drug is available for antimicrobial activity [8], which must be considered when examining the relationship between PK/PD parameters and in vivo activity [9]. A large number of studies have defined the PK/PD properties of the major classes of antibiotic, and have observed three patterns of activity $[10,11]$. The first is characterized by concentration-dependent killing and a prolonged post-antibiotic effect (PAE). Higher drug concentrations result in more rapid and extensive organism killing, and the peak/MIC $\left(\mathrm{C}_{\max } / \mathrm{MIC}\right)$ and/or the area under the concentration-time curve at $24 \mathrm{~h} / \mathrm{MIC}$ $\left(\mathrm{AUC}_{0-24} / \mathrm{MIC}\right)$ ratios are the best PK/PD parameters correlating with treatment efficacy. Dosing of drugs exhibiting this pattern of activity is optimized by the administration of large doses. Furthermore, a prolonged PAE allows the lengthening of dosing intervals (e.g. a once-daily dose regimen). This pattern is predictive of the activity of aminoglycosides, fluoroquinolones, metronidazole and daptomycin.

The second pattern is characterized by time-dependent killing and a minimal to moderate PAE. Extending the duration of exposure optimizes the antimicrobial activity. The time that free antimicrobial concentrations remain above the MIC $\left(T_{>M I C}\right)$ for the organism is the PK/PD index that correlates with bacterial killing and microbiological response. The shorter the drug elimination half-life, the more frequent the dose fractioning must be. In some circumstances the use of a continuous intravenous 
infusion, which maintains the $T_{>\text {MIC }}$ at $100 \%$, may be the most effective way of maximizing pharmacodynamic exposure, especially if higher $\mathrm{T}_{>\text {MIC }}$ are required [12]. The different classes of $\beta$-lactam (penicillins, cephalosporins, monobactams and carbapenems) exhibit this pattern of activity.

Concentration-independent killing and prolonged PAE characterize the final pattern of activity. Higher drug concentrations at most only slightly enhance organism killing, but produce prolonged suppression of organism regrowth. The goal of dosing is to optimize the amount of drug, and the $\mathrm{AUC}_{0-24} / \mathrm{MIC}$ ratio is the index most closely associated with efficacy. This is the pattern observed for glycopeptides, linezolid, quinupristindalfopristin, tetracyclines, clindamycin, azithromycin and the glycylcyclines.

A strong interrelationship (so-called colinearity) exists among the PK/PD indexes. With each increase in dose level, $\mathrm{C}_{\max }, \mathrm{AUC}_{0-24}$ and $\mathrm{T}_{>\mathrm{MIC}}$ will rise. Regarding the emergence of resistance, an increasing amount of data from in vitro and animal infection models have demonstrated a strict relationship between the magnitude of PK/PD parameters and the prevention of resistance, although data in humans are more limited [13].

\section{Critical illness and pharmacokinetic changes}

Critically ill patients with bloodstream infections include representatives of all age groups who have a range of organ dysfunctions related to severe acute illness that may complicate long-term illness. These factors, together with drug interactions and other therapeutic interventions (e.g. haemodynamically active drugs and continuous renal replacement therapies), may affect drug pharmacokinetics [14,15]. 
Variations in the extracellular fluid content and/or in renal or liver function are the most relevant and frequent pathophysiological mechanisms possibly affecting drug disposition in critically ill patients. Hydrophilic antimicrobials (e.g. $\beta$-lactams, aminoglycosides and glycopeptides) and renally excreted, moderately lipophilic antimicrobials (e.g. ciprofloxacin, gatifloxacin and levofloxacin) have to be considered at high risk of presenting substantial daily fluctuations in plasma concentration that may require repeated dosage adjustments. Hydrophilic antimicrobials exhibit a volume of distribution $\left(V_{d}\right)$ limited by the extracellular space, and their plasma and interstitial concentrations may drop dramatically because of substantial fluid extravasation to the interstitial space, known as third spacing. On the other hand, for lipophilic antimicrobials presenting larger $V_{d}$, the dilution of interstitial fluids is less relevant [16].

Several pathophysiological conditions may cause an increase of $V_{d}$ and dilution of antimicrobials in plasma and extracellular fluids so that an increase in dosage should be considered [16]. This may be especially true for hydrophilic concentration-dependent antimicrobials with a small $V_{d}$ (mainly aminoglycosides), which require loading doses at the start of therapy. The presence of an oedematous status, regardless of the underlying pathogenetic mechanism, plays a major role in altering the distribution of antimicrobials. Sepsis and trauma are the most common among the several causes of oedema. Higher dosages for most hydrophilic antimicrobials (either aminoglycosides or $\beta$-lactams) should therefore be considered to ensure therapeutic concentrations are maintained. Abundant intravenous fluid therapies, total parenteral nutrition, pleural effusion, mediastinitis, peritoneal exudates and ascites, by causing an increase in the extracellular compartment fluid, may lead to a significant increase in $V_{d}$ and the 
resulting dilution may justify higher dosages. In surgical patients, indwelling drainages may represent a pathway of antimicrobial loss and contribute to lower antimicrobial levels. Hypoalbuminaemia, a common condition in critically ill patients, may contribute to fluid extravasation and antimicrobial dilution by reducing plasma oncotic pressure, whereas the increase in the free fraction of drugs may increase their $V_{d}$. With reference to renally excreted highly albumin-bound antimicrobials (e.g. teicoplanin and ceftriaxone), the increase in free fraction caused by hypoalbuminaemia may promote not only more extensive distribution but also higher renal clearance.

In the intensive care unit (ICU) setting the use of haemodynamically active drugs (e.g. dopamine, dobutamine and furosemide) can modify renal blood flow and thereby glomerular filtration, tubular secretion rates and renal clearance. Extensive third-degree burns (>30-40\% body surface) cause enhanced renal clearance, with more relevant pathophysiological changes occurring beyond $48 \mathrm{~h}$, when the hypermetabolic phase usually begins. This period is frequently characterized by an increase in cardiac output leading to enhanced renal blood flow and, in turn, glomerular filtration rate, which may become significantly increased $[17,18]$. As a consequence, the renal clearance of most hydrophilic and moderately lipophilic antimicrobials is expected to increase significantly during the hypermetabolic phase. The same applies to the early phase of sepsis, which may cause an increase in cardiac output and renal blood flow [19]. Renal failure is also a common condition in the ICU setting.

Therapeutic drug monitoring may be of great value in all the clinical conditions described above, helping to optimize drug exposure in the individual patient. Several 
clinical trials have demonstrated its positive impact on clinical outcome and the cost of hospitalization [20].

\section{Glycopeptides}

The study of the pharmacodynamics of glycopeptides in animal models supports the concept that sustained higher concentrations or more frequent dosing can improve survival in animal models of infective endocarditis $[21,22]$. T>MIC was thus initially considered the most important PK/PD marker for efficacy. However, in a Streptococcus pneumoniae non-neutropenic mouse peritonitis model, Knudsen et al. demonstrated that both $\mathrm{T}_{>\mathrm{MIC}}$ and $\mathrm{C}_{\max }$ are of major importance for predicting the effect of single-dose glycopeptide treatment [23]. The same authors subsequently evaluated the effect of a wide spectrum of different treatment regimens with vancomycin and teicoplanin in an immunocompetent mouse peritonitis model with Staphylococcus aureus and S. pneumoniae as infective organisms [24]. The data showed that $\mathrm{C}_{\text {max-free }}$ was of major importance in the one- and two-dose trials, but this parameter alone could not explain the effects achieved in the multidose trials. In this setting, only $\mathrm{C}_{\text {max-free }}$ in combination with AUC/MIC for vancomycin or $\mathrm{T}_{>\text {MIC-free }}$ for teicoplanin was able to explain survival. In a subsequent review, Craig emphasized the role of AUC/MIC as predictive of efficacy not only for vancomycin but also for teicoplanin [11].

Several studies have emphasized the role of the AUC/MIC ratio as predictive of treatment efficacy. Hyatt et al. demonstrated that those patients treated with vancomycin monotherapy for enterococcal infections who achieved $\mathrm{AUC}_{0-24} / \mathrm{MIC}$ values $<125$ had a higher probability of clinical failure and selection of vancomycin-resistant Enterococcus faecium [25]. In a population of elderly hospitalized patients with lower 
respiratory tract infections caused by $S$. aureus and treated with vancomycin, MoiseBroder et al. showed that $\mathrm{AUC}_{0-24} / \mathrm{MIC}$ values predicted clinical and bacteriological outcomes, with higher clinical success rates in the subset of patients with $\mathrm{AUC}_{0-24} / \mathrm{MIC}$ values $>350$ (or approximately 400 for bacterial eradication) [26]. All patients in this study (both successes and failures) had $\mathrm{T}_{>\mathrm{MIC}}=100 \%$, establishing that vancomycin $\mathrm{T}_{>\text {MIC }}$ at the $100 \%$ target is not predictive of outcome.

In critically ill patients, the pharmacokinetics of vancomycin, like other antimicrobials, shows broad variability and a significant change in both clearance and the distribution volume [27]. Higher doses of vancomycin seem to be necessary in the ICU, even when the pathogens have MIC values typical of susceptible microorganisms, and therapeutic drug monitoring is strongly recommended, with the aim of optimizing drug exposure in the individual patient. In a recent retrospective study by Del Mar Fernández de Gatta Garcia et al., higher distribution volumes (nearly twice the quoted value of $0.72 \mathrm{~L} / \mathrm{kg}$ ) and different vancomycin clearance-creatinine clearance relationships were found in ICU patients [28]. Renal function, the APACHE score (Acute Physiology and Chronic Health Evaluation), age and serum albumin accounted for more than $65 \%$ of vancomycin clearance variability. According to PK/PD analysis, vancomycin standard dosages led to a $33 \%$ risk of not achieving the recommended $\mathrm{AUC}_{0-24} / \mathrm{MIC}$ breakpoint for $S$. aureus, possibly leading to an unfavourable clinical outcome. The results of Monte Carlo simulation revealed that doses of $3000 \mathrm{mg}$ or even $4000 \mathrm{mg}$ daily may be necessary to reach the highest probability of efficacy when susceptible $S$. aureus strains are involved in the infectious process; similar results were found for other Staphylococcus isolates. Regarding glycopeptide-intermediate S. aureus (GISA) strains, 
doses as high as $5000 \mathrm{mg} /$ day led to a maximum probability of a positive clinical outcome of only $80 \%$ for a value of 400 as the breakpoint. The results also point to the suitability of considering antimicrobial agents other than vancomycin when GISA strains are involved, as suggested by other authors [29].

With the aim of improving the results of vancomycin therapy, a variety of strategies such as higher doses, combination therapy and continuous infusion have been proposed. Continuous infusion might make treatment monitoring and adjustment easier and cheaper because vancomycin concentrations in serum are less variable and more sustained [30]. In a prospective multicenter randomized trial comparing critically ill patients with severe meticillin-resistant staphylococcal infections, continuous infusion of vancomycin resulted in therapeutic concentrations being achieved more quickly, less AUC variability between patients, fewer samples required to monitor treatment, and reduced 10-day antibiotic cost; clinical efficacy and safety were comparable to the intermittent infusion schedule [31]. AUC ${ }_{0-24} / \mathrm{MIC}$ values were not investigated in this study, meaning that no adjustment was made for organisms having different MIC values. Given the variation in $\mathrm{AUC}_{0-24} / \mathrm{MIC}$ that would result from the fourfold range in MIC values found in these patients $(0.5-2.0 \mathrm{mg} / \mathrm{L})$, it is not surprising that the $\mathrm{AUC}_{0-}$ ${ }_{24} / \mathrm{MIC}$ did not correlate with outcome, since dosage adjustments to target serum concentrations should make $\mathrm{AUC}_{0-24} / \mathrm{MIC}$ values similar in all patients (successes and failures) [26]. Di Filippo et al. observed more favourable clinical outcomes in patients with continuous infusion of vancomycin in terms of improved organ function and leukocyte response, but overall disease evolution was not altered [32], probably because the study sample was too small $(N=25)$. Data suggesting improved clinical 
cure and resolution of illness with continuous infusion of vancomycin in ICU patients are scarce. Nevertheless, a recent study by Rello et al. reported for the first time lower mortality rates among ICU patients with ventilator-associated pneumonia caused by oxacillin-resistant S. aureus receiving vancomycin in continuous infusion ( $25 \%$ vs. $55 \%)$ [33]. However, only a minority of the patients received continuous infusion of vancomycin $(N=16)$ and no detailed information on patients treated with continuous versus intermittent infusion was reported.

Finally, other authors have suggested that as vancomycin has a long elimination half-life (compared with $\beta$-lactams) and a prolonged Gram-positive antimicrobial effect, continuous infusion is unnecessary for most patients [34]. Larger and well-designed randomized trials are needed to clarify the clinical efficacy of this type of approach. The evidence to support advantages is very limited, as recently reported by the Infectious Diseases Society of America [35].

\section{Linezolid, quinupristin-dalfopristin, daptomycin and tigecycline}

\subsection{Linezolid}

This is the first member of a new class of antibacterial agents, the oxazolidinones, which act by inhibiting the formation of bacterial protein synthesis initiation complex, possibly by distorting the binding site for initiator tRNA [36]. It is a valid therapeutic alternative to glycopeptides against multiresistant Gram-positive strains such as staphylococci, streptococci and enterococci, which are particularly frequent in the ICU [37]. Linezolid is a time-dependent antimicrobial agent with a persistent antibiotic effect and the PK/PD indices $T_{>M I C}$ and AUC/MIC are important determinants of its efficacy in vitro and in vivo $[38,39]$. In several animal infection models, a $\mathrm{T}_{>\mathrm{MIC}}>40 \%$ significantly 
enhanced bacterial killing of pneumococci, and $A U C / M I C$ ratios of $48-147$ were necessary for a bacteriostatic effect $[38,38,40]$. Linezolid serum levels with $\mathrm{T}_{>\mathrm{MIC}}>50 \%$ for pathogens with MICs of 2-4 mg/L can be obtained in healthy volunteers by administration of $600 \mathrm{mg}$ every $12 \mathrm{~h}$ [41].

In critically ill patients Rayner et al. confirmed that both $\mathrm{T}_{>\mathrm{MIC}}$ and AUC/MIC are highly correlated with the probability of eradication and clinical cure within specific infection sites [38]. Higher success rates for linezolid may occur at AUC/MIC values of 80-120 for bacteraemia, lower respiratory tract infection and skin and skin structure infection. Chances of success in bacteraemia, lower respiratory tract infection and skin structure infection also appear to be higher when $\mathrm{T}_{>\mathrm{MIC}}>85 \%$. However, as mentioned above, alterations in pharmacokinetic parameters (especially volume of distribution and clearance) are common in critically ill patients and suboptimal serum and tissue concentrations may be achieved when drugs are administered at the standard dosage, with the risk of therapeutic failure and development of resistance.

Buerger et al. have recently demonstrated a high inter-individual variability in linezolid interstitial concentrations in patients with sepsis or septic shock, suggesting that a more frequent linezolid daily dosing scheme would be more appropriate in this subpopulation of patients [42]. In order to optimize the time-dependence of linezolid, the administration by continuous infusion has been proposed. In an in vivo model of endocarditis, Jacqueline et al. showed not only that continuous infusion was more effective than intermittent doses, but also that switching from intermittent dosing to continuous infusion (at the same daily dose) led to in vivo bactericidal activity [43]. Moreover, a recent trial by Adembri et al. compared the pharmacokinetic/ pharmacodynamic profile of linezolid 
administered by intermittent or continuous infusion in 16 critically ill septic patients [37]. In the intermittent infusion group, linezolid trough serum levels $\left(\mathrm{C}_{\min }\right)$ varied widely and were below the susceptibility breakpoint $(4 \mathrm{mg} / \mathrm{L})$ during the study period; in $50 \%$ of patients $\mathrm{C}_{\min }$ was $<1 \mathrm{mg} / \mathrm{L}$. In the continuous infusion group, mean linezolid serum levels were more stable and, starting from $6 \mathrm{~h}$, were significantly higher than $\mathrm{C}_{\min }$ levels observed in the intermittent infusion group and were always above the susceptibility breakpoint. Moreover, $T_{>M I C}>85 \%$ for the free drug was more frequent in the continuous infusion group. Finally, with continuous infusion it was possible to achieve AUC/MIC values of 80-120 more frequently than with intermittent infusion. No differences in clinical efficacy or microbiological eradication between the two regimens were observed, probably because of the small number of subjects, and no specific side effects due to continuous infusion were noted.

Since constant exposure of bacteria to linezolid levels just above the MIC has been shown to play a role in the development of in vitro resistance [44], another potential advantage of continuous infusion is that, by maintaining adequate serum levels, it may also reduce the phenomenon of resistance and may increase safety. Further studies with a larger number of patients are necessary to demonstrate the possible clinical benefit and safety of this administration modality.

\subsection{Quinupristin-dalfopristin}

This is a 30:70 mixture of two naturally occurring water-soluble streptogramin antimicrobials - pristinamycin IA (quinupristin: RP 57669), a peptidic macrolactone, and pristinamycin IIA (dalfopristin: RP 54476), a polyunsaturated macrolactone [45]. They demonstrate synergistic activity against a wide variety of Gram-positive organisms, 
including meticillin-resistant Staphylococcus aureus (MRSA) and vancomycin-resistant E. faecium [46-48]. Activity against most strains of Enterococcus faecalis is lacking [49]. Individually the antibiotics are bacteriostatic but, in combination, bactericidal activity can be observed against staphylococci and streptococci, although not against $E$. faecium [50]. In vitro studies suggest that quinupristin-dalfopristin exhibits time-dependent killing but produces prolonged persistent effects against Gram-positive bacteria at concentrations above the MIC [48], allowing extended administration intervals of $8 \mathrm{~h}$, despite the short half-lives of the two compounds $(<1 \mathrm{~h})$.

There are relatively few data to define the PK/PD parameter that predicts activity for quinupristin-dalfopristin. In vivo data using the neutropenic thigh model against $S$. aureus and S. pneumoniae showed $\mathrm{AUC}_{0-24} / \mathrm{MIC}$ to be the best predictor of response [51]. Other investigators have suggested that minimum bactericidal concentration may be a more appropriate denominator for pharmacodynamic outcomes [52-53]. Due to the multiple active components of pharmacokinetic models, the appropriate PK/PD handling of quinupristin-dalfopristin is not easy. The observation that bioassay results may approximate the additive activity of each parent compound with its active metabolites has led to the suggestion of combining the pharmacokinetic parameters of all high performance liquid chromatography-measured compounds.

Adding the reported AUCs of all known active components (quinupristin, dalfopristin, cysteine-quinupristin, glutathione-quinupristin and pristinamycin IIA) from the selective bioassays produces a combined steady-state AUC over the administration interval of approximately $16-17 \mathrm{mg} \cdot \mathrm{h} / \mathrm{L}$ [54]. This corresponds to a total drug by $\mathrm{AUC}_{24}$ of approximately $34 \mathrm{mg} \cdot \mathrm{h} / \mathrm{L}$ and $50 \mathrm{mg} \cdot \mathrm{h} / \mathrm{L}$ for the $7.5 \mathrm{mg} / \mathrm{kg}$ every $12 \mathrm{~h}$ and $7.5 \mathrm{mg} / \mathrm{kg}$ 
every $8 \mathrm{~h}$ regimens, respectively. For sensitive pathogens with a MIC of $1 \mathrm{mg} / \mathrm{L}$, a $24 \mathrm{~h}$ total drug AUC/MIC ratio of 34-50 would be obtained. The effect of protein binding on these parameters may further reduce these ratios, as only free drug is active. However, since the difference in protein binding $(55-78 \%$ for quinupristin and $11-26 \%$ for dalfopristin) of the two compounds is consistent, the magnitude of the PK/PD parameters based on free drug is not applicable for clinical use.

Thus, although AUC/MIC has been suggested as a marker for efficacy of quinupristindalfopristin, the most clinically effective value of this ratio has not been identified and human studies correlating PK/PD parameters with clinical outcomes are lacking [55].

\subsection{Daptomycin}

Daptomycin is a cyclic lipopeptide antimicrobial agent that only has activity against Gram-positive organisms. It is principally used to treat vancomycin-resistant enterococci, MRSA and glycopeptide-intermediate and -resistant $S$. aureus. [56]. It has been approved for the treatment of skin and soft tissue infections and $S$. aureus bloodstream infections, including right-sided endocarditis $[57,58]$. It was not successful in trials of community-acquired pneumonia and it has subsequently been shown that daptomycin is inactivated by surfactant, rendering it unable to kill bacteria in the alveoli $[59,60]$.

Daptomycin has a unique mechanism of action; it binds to bacterial membranes and causes rapid depolarization of membrane potential, resulting in inhibition of protein, DNA and RNA synthesis, leading to bacterial cell death [61]. It is characterized by rapid concentration-dependent killing and a prolonged antibiotic effect against $S$. aureus and enterococci $[62,63]$. In murine neutropenic thigh models of $S$. aureus meticillin- 
susceptible [64] or meticillin-resistant strains [65] infection, the AUC/MIC ratio has been correlated with outcome. In another neutropenic murine thigh infection model, Safdar et al. demonstrated that both the $A U C / M C_{0-24}$ ratio and the $C_{\max } / \mathrm{MIC}$ ratio were strong predictors of in vivo efficacy [66]. In vitro pharmacodynamic studies have shown that a range of free AUC/MIC ratios (16.5-189) was associated with $80 \%$ maximum effect for various Staphylococcus and Enterococcus spp. [67]. In general, however, lower AUC/MIC exposures are required to achieve static or $80 \%$ maximum effects for enterococci compared with staphylococci [68]. Over a $24 \mathrm{~h}$ period, free daptomycin concentrations averaging 1-2 times the MIC are needed for a bacteriostatic effect and 2-4 times the MIC for $>99 \%$ killing $[69,70]$.

Due to daptomycin's concentration dependence, a once-daily dosing regimen has been proposed to optimize its pharmacodynamic properties and has demonstrated an improved safety profile compared with that of the initially evaluated twice-daily regimen [71-75]. A Monte Carlo prediction model analysis was conducted to determine if AUC/MIC targets could be achieved in a clinical setting [74]. An AUC/MIC ratio of 189 for the free drug generated maximum-kill $E D_{80}$ (the effective doses required to achieve $80 \%$ kill) against MRSA and E. faecium isolates tested. The Monte Carlo simulations predicted the probability of achieving a free drug AUC/MIC ratio of 189 to be $80.4 \%$, $91.06 \%$ and $95.64 \%$ for doses of 4,6 and $8 \mathrm{mg} / \mathrm{kg}$ once per day.

Cha et al. used an in vitro pharmacodynamic model with simulated endocardial vegetations incorporating protein to simulate regimens of daptomycin at 6 and $8 \mathrm{mg} / \mathrm{kg} /$ day and vancomycin at $1 \mathrm{~g}$ every $12 \mathrm{~h}$ against meticillin-resistant $S$. aureus and Staphylococcus epidermidis, glycopeptide-intermediate S. aureus and S. epidermidis, 
and vancomycin-resistant E. faecium [75]. Both daptomycin regimens achieved greater than $99.9 \%$ kill by $8 \mathrm{~h}$ and demonstrated greater bacterial reduction than vancomycin against all tested isolates at 24, 48 and $72 \mathrm{~h}$. Higher daptomycin dosage (i.e. $6 \mathrm{mg} / \mathrm{kg}$ intravenously once per day) is currently approved by the US Food and Drug Administration for $S$. aureus bloodsteam infections, including patients with right-sided endocarditis [76].

At present there are no pharmacokinetic studies in ICU patients. Therefore monitoring of the drug for efficacy and for safety is recommended in these patients.

\subsection{Tigecycline}

This derivative of minocycline is the first of a new class of antimicrobials known as glycylcyclines. Tigecycline not only has in vitro activity against MRSA and vancomycinresistant $E$. faecium, but also common Gram-negative aerobes, atypical pathogens and anaerobic pathogens, with the exception of Pseudomonas aeruginosa and Proteus spp. [77-81]. As with other tetracycline derivatives, tigecycline binds to the $30 \mathrm{~S}$ ribosomal subunit, inhibiting protein synthesis and bacterial growth. The presence of a modified side chain on tigecycline, with respect to minocycline, circumvents resistance mechanisms that plague tetracycline and other antibiotics in this class. Tigecycline has been evaluated for the treatment of complicated skin and skin structure infections, complicated intra-abdominal infections, and pneumonia [82-83]. In vitro, tigecycline is characterized by time-dependent activity against $S$. pneumoniae, Haemophilus influenzae, and Neisseria gonorrhoeae [84-86]. Due to its prolonged PAE and relatively long half-life (approximately $40 \mathrm{~h}$ ), tigecycline's AUC/MIC ratio is the PK/PD index most likely to be predictive of its clinical and microbiological efficacy [87-90]. In a S. aureus 
neutropenic mouse thigh infection model, values of $15-20$ for $\mathrm{AUC}_{0-24} / \mathrm{MIC}$ were associated with stasis to a $90 \%$ reduction in bacterial burden [89]. Recent studies by Meagher et al. involved hospitalized patients with complicated skin and soft tissue infections enrolled in clinical trials and treated with tigecycline [91]. Multivariate logistic regression analyses identified $\mathrm{AUC}_{0-24} / \mathrm{MIC}$ as being predictive of microbiological response to therapy. When considering S. aureus and/or group A streptococcal infections, $\mathrm{AUC}_{0-24}: \mathrm{MIC}$ values $>17.9$ were associated with $100 \%$ of patients having a microbiological success, whereas patients with $\mathrm{AUC}_{0-24}$ :MIC values $<17.9$ had only a $50 \%$ response; a similar exposure-response relationship was also observed for clinical outcome. The breakpoints identified above reflect those obtained in patients with complicated skin and skin structure infections and should not be extrapolated to other infections [91-93]. The relationship between tigecycline exposure and response has also been evaluated in patients with complicated intra-abdominal infections [92]. Ninetyfour percent of patients with an AUC/MIC ratio $>6.96$ had resolution of signs and symptoms of infection and required no further antimicrobial therapy. Compared with those with an AUC:MIC ratio $\leq 6.96$, these patients were 5.7 times more likely to have a clinical response and 10 times more likely to have a microbiological response. Further studies are needed to determine whether the AUC/MIC ratio is a reliable pharmacodynamic parameter for predicting the outcome in patients receiving tigecycline

\section{Conclusion}

Patients in ICUs are at high risk of developing bacterial infections with a high mortality rate. Every effort should be undertaken to minimize the rate of nosocomial infection by 
appropriate infection control programmes, and the microbiological laboratory should be able to provide regularly updated reviews of resistance patterns for the most important pathogens isolated in the ICU [94]. Optimization of antimicrobial therapy is mandatory $[95,96]$.

The aim of anti-infective therapy is to administer a dose of drug that will have an acceptably high probability of attaining the desired therapeutic effect while having an acceptably low probability of concentration-related toxicity. PK/PD relationships are the major determinants of in the vivo efficacy of antimicrobial agents and allow optimization of the dosage regimen to improve the outcome and reduce the selection of resistant mutants. Although there are relatively few clinical studies available in the ICU setting to deliver the proof of concept, several in vitro data and animal studies provide good evidence for the beneficial role of optimizing the PK/PD relationship for most of the antibiotics used in clinical practice. The MIC of an antimicrobial agent against the infecting pathogen is a very important parameter in this relationship and it is the responsibility of the microbiology laboratory to deliver a qualified and quality-assured estimate of this value; the clinician may then consider this information when choosing the correct antimicrobial treatment. In the ICU setting, high pharmacokinetic variability, together with the variability of microbe susceptibility, led to poor predictability of PK/PD markers based on general population data [96].

Once we have a goal of therapy, the first step is to define the drug dose that has a high probability of delivering the desired target. The Monte Carlo simulation allows the calculation of the proportion of patients obtaining a specific degree of drug exposure. Afterward, the exposures are corrected for protein binding and the fraction of simulated 
subjects who attained the pharmacodynamic target is calculated for each MIC in the distribution. Overall target attainment is then calculated by taking the product of the target attainment at a specific MIC and the fraction of organisms in the distribution at that MIC. All products are then summed, giving a weighted average target attainment rate that takes into account the variability in MICs as well as the variability in pharmacokinetic parameters across a specific population of patients. Finally, Bayesian estimation allows patient-specific estimation of drug exposure. This estimation process explicitly balances information about the specific patient (the drug concentrations obtained from that patient) with prior knowledge about how a specific patient population handles a particular drug. In this way, the best point estimates of the pharmacokinetic parameter values for each patient in the data set can be obtained, thus allowing calculation of the $\mathrm{C}_{\max } / \mathrm{MIC}$ ratio, $\mathrm{AUC} / \mathrm{MIC}$ ratio or $\mathrm{T}_{>\mathrm{MIC}}$.

Several studies have confirmed that a Bayesian method may be helpful for individualizing dosing regimens of antimicrobials in the ICU setting $[97,98]$. Furthermore, since the physiology of ICU patients may change over a relatively short period, ongoing evaluation of sickness severity and therapeutic drug monitoring are strongly recommended to allow timely adjustment of antibacterial dosing. This should not only be achieved for antimicrobials that have plasma concentrations routinely monitored, but for all antimicrobials.

Every effort should be undertaken by clinicians, microbiologists and pharmacologists to improve the microbiological diagnosis and the PK/PD correlation. These efforts may result in a better clinical outcome and a reduction in antibiotic resistance levels and economic costs. 
Funding: The author received an honorarium for writing this article. The funds for the honorarium were provided by Novartis AG, Switzerland and were handled by the organizing committee of the 4th European Conference on Bloodstream Infections for the publication of this supplement.

Competing interests: There are no potential conflicts of interest to disclose in the context of this article.

Ethical approval: Not required. 


\section{References}

[1] Vincent JL, Bihari DJ, Suter PM, Bruining HA, White J, Nicolas-Chanoin MH, et al. The prevalence of nosocomial infection in intensive care units in Europe. Results of the European Prevalence of Infection in Intensive Care (EPIC) Study. EPIC International Advisory Committee. JAMA 1995;274:639-44.

[2] Weber DJ, Raasch R, Rutala WA. Nosocomial infections in the ICU: the growing importance of antibiotic-resistant pathogens. Chest 1999;115(Suppl 3):34-41S.

[3] Vincent JL. Nosocomial infections in adult intensive care units. Lancet 2003;361:2068-77.

[4] Paterson DL. Restrictive antibiotic policies are appropriate in intensive care units. Crit Care Med 2003;31(Suppl 1):S25-28.

[5] Kollef $\mathrm{MH}$. Optimizing antibiotic therapy in the intensive care unit setting. Critical Care 2001;5:189-95.

[6] Scaglione F, Paraboni L. The influence of pharmacokinetic/pharmacodynamic (PK/PD) of antibacterials in their dosing regimens selection. Expert Rev Anti Infect Ther 2006;4:479-90.

[7] Drusano GL Antimicrobial pharmacodynamics: critical interactions of 'bug and drug'. Nat Rev Microbiol 2004;2:289-300.

[8] Craig WA, Suh B. Protein binding and the antimicrobial effects: methods for the determination of protein binding. In: Lorian V, editor. Antibiotics in laboratory medicine. Baltimore: Williams and Wilkins; 1991, pp. 367-402. 
[9] Andes D, Craig WA. Animal model pharmacokinetics and pharmacodynamics: a critical review. Int J Antimicrob Agents 2002;19:261-8.

[10] Craig WA. Does the dose matter? Clin Infect Dis 2001;33(Suppl):S233-S237.

[11] Craig WA. Basic pharmacodynamics of antibacterials with clinical applications to the use of $\beta$-lactams, glycolpeptides and linezolid. Infect Dis Clin North Am 2003;17:479-501.

[12] Pea F, Viale P. The antimicrobial therapy puzzle: Could pharmacokineticpharmacodynamic relationships be helpful in addressing the issue of appropriate pneumonia treatment in critically ill patients? Clin Infect Dis 2006;42:1764-71.

[13] Drusano GL. Prevention of resistance: A goal for dose selection of antimicrobial agents. Clin Infect Dis 2003;36(Suppl 1):S42-S50.

[14] Wagenlehner FME, Weidner W, Naber KG. Pharmacokinetic characteristics of antimicrobials and optimal treatment of urosepsis. Clin Pharmacokinet 2007;46:291305.

[15] Pea F, Furlanut M. Pharmacokinetic aspects of treating infections in the intensive care unit: focus on drug interactions. Clin Pharmacokinet 2001;40:833-68.

[16] Pinder M, Bellomo R, Lipman J. Pharmacological principles of antibiotic prescription in the critically ill. Anaesth Intensive Care 2002;30:134-44.

[17] Weinbren MJ. Pharmacokinetics of antibiotics in burn patients. J Antimicrob Chemother 1999;44:319-27

[18] Jaehde U, Sorgel F. Clinical pharmacokinetics in patients with burns. Clin Pharmacokinet 1995;29:15-28. 
[19] Roberts JA, Lipman J. Antibacterial dosing in intensive care: pharmacokinetics, degree of disease and pharmacodynamics of sepsis. Clin Pharmacokinet 2006;45:75573.

[20] Bartal C, Danon A, Schlaeffer F, Reisenberg K, Alkan M, Smoliakov R, et al. Pharmacokinetic dosing of aminoglycosides: a controlled trial. Am J Med 2003;114:194-8.

[21] Contrepois A, Joly V, Abel L, Pangon B, Vallois JM, Carbon C. The pharmacokinetics and extravascular diffusion of teicoplanin in rabbits and comparative efficacy with vancomycin in an experimental endocarditis model. J Antimicrob Chemother 1988;21:621-31.

[22] Chambers HF, Kennedy S. Effect of dosage, peak and trough concentrations in serum, protein binding and bactericidal rate of efficacy of teicoplanin in a rabbit model of endocarditis. Antimicrob Agents Chemother 1990;34:510-14.

[23] Knudsen JD, Fuursted K, Espersen F, Frimodt-Moller N. Activities of vancomycin and teicoplanin against penicillin-resistant pneumococci in vitro and in vivo and correlation to pharmacokinetic parameters in the mouse peritonitis model. Antimicrob Agents Chemother 1997;41:1910-15.

[24] Knudsen JD, Fuursted K, Raber S, Espersen F, Frimodt-Møller N.

Pharmacodynamics of glycopeptides in the mouse peritonitis model of Streptococcus pneumoniae or Staphylococcus aureus infection. Antimicrob Agents Chemother 2000;44:1247-54. 
[25] Hyatt JM, McKinnon PS, Zimmer GS, Schentag JJ. The importance of pharmacokinetic/pharmacodynamic surrogate markers to outcome: focus on antibacterial agents. Clin Pharmacokinet 1995;28:143-60.

[26] Moise-Broder PA, Forrest A, Birmingham MC, Schentag JJ. Pharmacodynamics of vancomycin and other antimicrobials in patients with Staphylococcus aureus lower respiratory tract infections. Clin Pharmacokinet 2004;43:925-42.

[27] Polard E, Le Bouquin V, Le Corre P, Kérebel C, Trout H, Feuillu A, et al. Non steady state and steady state PKS Bayesian forecasting and vancomycin pharmacokinetics in ICU adult patients. Ther Drug Monit 1999;21:395-403.

[28] Del Mar Fernández de Gatta Garcia M, Revilla N, Calvo MV, Domínguez-Gil A, Sánchez Navarro A. Pharmacokinetic/pharmacodynamic analysis of vancomycin in ICU patients. Intensive Care Med 2007;33:279-85.

[29] Schentag JJ Antimicrobial management strategies for Gram-positive bacterial resistance in the intensive care unit. Crit Care Med 2001;29(Suppl 4):S100-S107. [30] Byl B, Clevenbergh P, Jacobs F, Struelens MJ, Zech F, Kentos A, et al: Impact of infectious diseases specialists and microbiological data on the appropriateness of antimicrobial therapy for bacteremia. Clin Infect Dis 1999;29:60-6.

[31] Wysocki M, Delatour F, Faurisson F, Rauss A, Pean Y, Misset B, et al: Continuous versus intermittent infusion of vancomycin in severe staphylococcal infections: Prospective multicenter randomized study. Antimicrob Agents Chemother $2001 ; 45: 2460-7$. 
[32] Di Filippo A, De Gaudio AR, Novelli A, Paternostro E, Pelagatti C, Livi P, et al:

Continuous infusion of vancomycin in methicillin-resistant staphylococcus infection. Chemotherapy 1998;44:63-8.

[33] Rello J, Sole-Violan J, Sa-Borges M, Garnacho-Montero J, Muñoz E, Sirgo G, et al. Pneumonia caused by oxacillin-resistant Staphylococcus aureus treated with glycopeptides. Crit Care Med 2005;33:1983-7.

[34] Slavik RS, Jewesson PJ. Selecting antibacterials for outpatient parenteral antimicrobial therapy: pharmacokinetic-pharmacodynamic consideration. Clin Pharmacokinet 2003;42:793-817.

[35] Rybak MJ, Lomaestro BM, Rotscahfer JC, Moellering RC, Craig AW, Billeter M, et al. Vancomycin therapeutic guidelines: a summary of consensus recommendations from the Infectious Diseases Society of America, the American Society of Health-System Pharmacists, and the Society of Infectious Diseases Pharmacists. Clin Infect Dis 2009;49:325-7.

[36] Stalker DJ, Jungbluth GL. Clinical pharmacokinetics of linezolid, a novel oxazolidinone antibacterial. Clin Pharmacokinet 2003;42:1129-40.

[37] Adembri A, Fallani S, Cassetta MI, Arrigucci S, Ottaviano A, Pecile P, et al. Linezolid pharmacokinetic/pharmacodynamic profile in critically ill septic patients: intermittent versus continuous infusion. Int J Antimicrob Agents 2008;31:122-9. [38] Rayner CR, Forrest A, Meagher AK, Birmingham MC, Schentag JJ. Clinical pharmacodynamics of linezolid in seriously ill patients treated in a compassionate use program. Clin Pharmacokinet 2003;42:1411-23. 
[39] MacGowan AP. Pharmacokinetic and pharmacodynamic profile of linezolid in healthy volunteers and patients with Gram-positive infections. J Antimicrob Chemother 2003;51(Suppl 2):ii17-25.

[40] Gentry-Nielsen MJ, Olsen KM, Preheim LC. Pharmacodynamic activity and efficacy of linezolid in a rat model of pneumococcal pneumonia. Antimicrob Agents Chemother 2002;46:1345-51.

[41] Dailey CF, Dileto-Fang CL, Buchanan LV, Oramas-Shirey MP, Batts DH, Ford CW, et al. Efficacy of linezolid in treatment of experimental endocarditis caused by methicillin-resistant Staphylococcus aureus. Antimicrob Agents Chemother $2001 ; 45: 2304-8$.

[42] Buerger C, Plock N, Dehghanyar P, Joukhadar C, Kloft C. Pharmacokinetics of unbound linezolid in plasma and tissue interstitium of critically ill patients after multiple dosing using microdialysis. Antimicrob Agents Chemother 2006;50:2455-63.

[43] Jacqueline C, Batard E, Perez L, Boutoille D, Hamel A, Caillon J, et al. In vivo efficacy of continuous infusion versus intermittent dosing of linezolid compared to vancomycin in a methicillin-resistant Staphylococcus aureus rabbit endocarditis model. Antimicrob Agents Chemother 2002;46:3706-11.

[44] Boak LM, Rayner CR, Nation RL. Pharmacokinetic/pharmacodynamic factors influencing emergence of resistance to linezolid in an in vitro model. Antimicrob Agents Chemother 2007;51:1287-92.

[45] Rybak MJ, Houlihan HH, Mercier RC, Kaatz GW. Pharmacodynamics of RP 59500 (quinupristin-dalfopristin) administered by intermittent versus continuous infusion 
against Staphylococcus aureus-infected fibrin-platelet clots in an in vitro infection model. Antimicrob Agents Chemother 1997;41:1359-63.

[46] Jones RN, Ballow CH, Biedenbach DJ, Deinhart JA, Schentag JJ. Antimicrobial activity of quinupristin-dalfopristin (RP 59500, Synercid) tested against over 28,000 recent clinical isolates from 200 medical centers in the United States and Canada. Diagn Microbiol Infect Dis 1998;31:437-51.

[47] Hershberger E, Aeschlimann JR, Moldovan T, Rybak MJ. Evaluation of bactericidal activities of LY333328, vancomycin, teicoplanin, ampicillin-sulbactam, trovafloxacin, and RP59500 alone or in combination with rifampin or gentamicin against different strains of vancomycin-intermediate Staphylococcus aureus by time-kill curve methods. Antimicrob Agents Chemother 1999;43:717-21.

[48] Lamb HM, Figgitt DP, Faulds D. Quinupristin/dalfopristin: a review of its use in the management of serious Gram-positive infections. Drugs 1999;58:1061-97.

[49] Bearden DT. Clinical pharmacokinetics of quinupristin/dalfopristin. Clin Pharmacokinet 2004;43:239-52.

[50] Eliopoulos GM: Quinupristin-dalfopristin and linezolid: evidence and opinion. Clin Infect Dis 2003;36:473-81.

[51] Craig W, Ebert S. Pharmacodynamic activities of RP 59500 in an animal infection model. In: Abstracts of 33rd Interscience Conference on Antimicrobial Agents and Chemotherapy; 17-20 Oct 1993; New Orleans, Abstract 470.

[52] Fantin B, Leclercq R, Merle Y, Saint-Julien L, Veyrat C, Duval J, et al. Critical influence of resistance to streptogramin B-type antibiotics on activity of RP 59500 
(quinupristin-dalfopristin) in experimental endocarditis due to Staphylococcus aureus. Antimicrob Agents Chemother 1995;39:400-5.

[53] Aeschlimann JR, Rybak MJ. Pharmacodynamic analysis of the activity of quinupristin-dalfopristin against vancomycin-resistant Enterococcus faecium with differing MBCs via time-kill-curve and postantibiotic effect methods. Antimicrob Agents Chemother 1998;42:2188-92.

[54] Bearden DT. Clinical pharmacokinetics of quinupristin/dalfopristin. Clin Pharmacokinet 2004;43:239-52.

[55] Carbon C. Pharmacodynamics of macrolides, azalides, and streptogramins: effect on extracellular pathogens. Clin Infect Dis 1998;27:28-32.

[56] Paterson DL. Clinical experience with recently approved antibiotics. Curr Opin Pharmacol 2006;6:486-90.

[57] Arbeit RD, Maki D, Tally FP, Campanaro E, Eisenstein BI. The safety and efficacy of daptomycin for the treatment of complicated skin and skin-structure infections. Clin Infect Dis 2004;38:1673-81.

[58] Fowler VG Jr, Boucher HW, Corey GR, Abrutyn E, Karchmer AW, Rupp ME, et al. Daptomycin versus standard therapy for bacteremia and endocarditis caused by Staphylococcus aureus. N Engl J Med 2006;355:653-65.

[59] Micek ST. Alternatives to vancomycin for the treatment of methicillin-resistant Staphylococcus aureus infections. Clin Infect Dis 2007;45(Suppl 3):S184-190. 
[60] Silverman JA, Mortin LI, Vanpraagh AD, Li T, Alder J: Inhibition of daptomycin by pulmonary surfactant: in vitro modeling and clinical impact. J Infect Dis 2005;191:2149_ 52.

[61] Straus SK, Hancock REW. Mode of action of the new antibiotic for Gram-positive pathogens daptomycin: comparison with cationic antimicrobial peptides and lipopeptides. Biochim Biophys Acta 2006;1758:1215-23.

[62] Fenton C, Keating GM, Curran MP. Daptomycin. Drugs 2004;64:445-55.

[63] Akins R, Rybak MJ. Bactericidal activities of two daptomycin regimens against clinical strains of glycopeptide intermediate-resistant Staphylococcus aureus, vancomycin-resistant Enterococcus faecium, and methicillin-resistant Staphylococcus aureus isolates in an in vitro pharmacodynamic model with simulated endocardial vegetations. Antimicrob Agents Chemother 2001;45:454-9.

[64] Louie A, Kaw P, Liu W, Jumbe N, Miller MH, Drusano GL. Pharmacodynamics of daptomycin in a murine thigh model of Staphylococcus aureus infection. Antimicrob Agents Chemother 2001;45:845-51.

[65] Dandekar PK, Tessier PR, Williams P, Nightingale CH, Nicolau DP. Pharmacodynamic profile of daptomycin against Enterococcus species and methicillinresistant Staphylococcus aureus in a murine thigh infection model. J Antimicrob Chemother 2003;52:405-11.

[66] Safdar N, Andes D, Craig WA. In vivo pharmacodynamic activity of daptomycin. Antimicrob Agents Chemother 2004;48:63-8. 
[67] Cha R, Grucz RG Jr, Rybak MJ. Daptomycin dose-effect relationship against resistant Gram-positive organisms. Antimicrob Agents Chemother 2003;47:1598-603. [68] Lee SY, Fan HW, Kuti JL, Nicolau DP. Update on daptomycin: the first approved lipopeptide antibiotic. Expert Opin Pharmacother 2006;7:1381-97.

[69] French GL. Bactericidal agents in the treatment of MRSA infections - the potential role of daptomycin. J Antimicrob Chemother 2006;58;1107-17.

[70] Oleson FB Jr, Berman CL, Kirkpatrick JB, Regan KS, Lai JJ, Tally FP. Once-daily dosing in dogs optimizes daptomycin safety. Antimicrob Agents Chemother 2000;44:2948-53.

[71] Tedesco KL, Rybak MJ. Daptomycin. Pharmacotherapy 2004;24:41-57.

[72] Tally FP, Zeckel M, Wasilewski, MM, Carini C, Berman, CL, Drusano, GL. Daptomycin: a novel agent for Gram-positive infections. Expert Opin Invest Drugs 1999;8:1223-38.

[73] Dvorchik BH, Brazier D, DeBruin MF, Arbeit RD. Daptomycin pharmacokinetics and safety following administration of escalating doses once daily to healthy subjects. Antimicrob Agents Chemother 2003;47:1318-23.

[74] Rybak MJ, McKinnon P, Cha R, Dvorchik BH. Daptomycin pharmacodynamics (PD) versus MRSA at various doses as assessed by a Monte Carlo prediction model. In: Program and abstracts of the 41st Interscience Conference on Antimicrobial Agents and Chemotherapy; 16-19 December 2001; Chicago, IL. Washington, DC: American Society for Microbiology; 2001, A-2195. 
[75] Cha R, Rybak MJ. Daptomycin against multiple drug-resistant Staphylococcus and Enterococcus isolates in an in vitro pharmacodynamic model with simulated endocardial vegetations. Diagn Microbiol Infect Dis 2003;47:539-46.

[76] Drew RH. Emerging options for treatment of invasive, multidrug-resistant Staphylococcus aureus infections. Pharmacotherapy 2007;27:227-49.

[77] Bradford PA, Weaver-Sands DT, Petersen PJ. In vitro activity of tigecycline against isolates from patients enrolled in phase 3 clinical trials of treatment for complicated skin and skin-structure infections and complicated intra-abdominal infections. Clin Infect Dis 2005;41(Suppl 5):S315-32.

[78] Milatovic D, Schmitz FJ, Verhoef J, Fluit AC. Activities of the glycylcycline tigecycline (GAR-936) against 1,924 recent European clinical bacterial isolates. Antimicrob Agents Chemother 2003;47:400-404.

[79] Cercenado E, Cercenado S, Gomez JA, Bouza E. In vitro activity of tigecycline (GAR-936), a novel glycylcycline, against vancomycin-resistant enterococci and staphylococci with diminished susceptibility to glycopeptides. J Antimicrob Chemother 2003;52:138-9.

[80] Kitzis MD, Ly A, Goldstein FW. In vitro activities of tigecycline (GAR-936) against multidrug-resistant Staphylococcus aureus and Streptococcus pneumoniae. Antimicrob Agents Chemother 2004;48:366-7.

[81] Rose WE, Rybak MJ. Tigecycline: first of a new class of antimicrobial agents. Pharmacotherapy 2006;26:1099-110. 
[82] Ellis-Grosse EJ, Babinchak T, Dartois N, Rose G, Loh E. The efficacy and safety of tigecycline in the treatment of skin and skin-structure infections: results of 2 double-blind phase 3 comparison studies with vancomycin-aztreonam.

Clin Infect Dis 2005;41(Suppl 5):S341-53.

[83] Babinchak T, Ellis-Grosse E, Dartois N, Rose GM, Loh E. The efficacy and safety of tigecycline for the treatment of complicated intra-abdominal infections: analysis of pooled clinical trial data. Clin Infect Dis 2005;41(Suppl 5):S354-67.

[84] Petersen PJ, Jacobus NV, Weiss WJ, Sum PE, Testa RT. In vitro and in vivo antibacterial activities of a novel glycylcycline, the 9-t-butylglycylamido derivative of minocycline (GAR-936). Antimicrob Agents Chemother 1999;43:738-44.

[85] Petersen PJ, Labthavikul P, Jones $\mathrm{CH}$, Bradford PA. In vitro antibacterial activities of tigecycline in combination with other antimicrobial agents determined by chequerboard and time-kill analysis. J Antimicrob Chemother 2006;57:573-6.

[86] Ziglam H. Daptomycin and tigecycline: a review of clinical efficacy in the antimicrobial era. Expert Opin Pharmacother 2006;8:2279-92.

[87] Meagher AK, Ambrose PG, Grasela TH, Ellis-Grosse EJ. The pharmacokinetic and pharmacodynamic profile of tigecycline. Clin Infect Dis 2005;41(Suppl 5):S333-40.

[88] Agwuh KN, MacGowan A. Pharmacokinetics and pharmacodynamics of the tetracyclines including glycylcyclines. J Antimicrob Chemother 2006;58:256-65.

[89] Van Ogtrop ML, Andes D, Stamstad TJ, et al. In vivo pharmacodynamic activities of two glycylcyclines (GAR-936 and WAY 152,288) against various Gram-positive and Gram-negative bacteria. Antimicrob Agents Chemother 2000;44:943-9. 
[90] Meagher AK, Ambrose PG, Grasela TH, Ellis-Grosse EJ.

Pharmacokinetic/pharmacodynamic profile for tigecycline-a new glycylcycline antimicrobial agent. Diagn Microbiol Infect Dis 2005;52:165-71.

[91] Meagher AK, Passarell JA, Cirincione BB, Van Wart SA, Liolios K, Babinchak T, et al. Exposure-response analyses of tigecycline efficacy in patients with complicated skin and skin-structure infections. Antimicrob Agents Chemother 2007;51:1939-45.

[92] Passarell JA, Meagher AK, Liolios K, Cirincione BB, Van Wart SA, Babinchak T, et al. Exposure-response analyses of tigecycline efficacy in patients with complicated intra-abdominal infections. Antimicrob Agents Chemother 2008;52:204-10.

[93] Doan TL, Fung HB, Mehta D, Riska PF. Tigecycline: a glycylcycline antimicrobial agent. Clin Ther 2006;28:1079-106.

[94] Glück T, Opal SM. Advances in sepsis therapy. Drugs 2004;64:837-59.

[95] Lode H. Management of serious nosocomial bacterial infections: do current therapeutic options meet the need? Clin Microbiol Infect 2005;11:778-87.

[96] Scaglione F. Can PK/PD be used in everyday clinical practice. Int J Antimicrob Agents 2002;19:349-53.

[97] Fernandez de Gatta MM, Fruns I, Dominguez-Gil A. Individualizing vancomycin dosing regimens: an evaluation of two pharmacokinetic dosing programs in critically ill patients. Pharmacotherapy 1994;14:196-201.

[98] Gauthier T, Lacarelle B, Marre F, Villard PH, Catalin J, Durand A. Predictive performance of two software packages (USC*- PACK PC and Abbott PKS system) for 
the individualization of amikacin dosage in intensive care unit patients. Int $\mathrm{J}$ Biomed Comput 1994;36:131-4. 
[Figure legend]

Fig. 1. Mortality impact of inadequate antimicrobial therapy in bacteraemia. ${ }^{a}$ Resistant Gram-negative microorganisms; ${ }^{b}$ Community-acquired bacteraemia. 


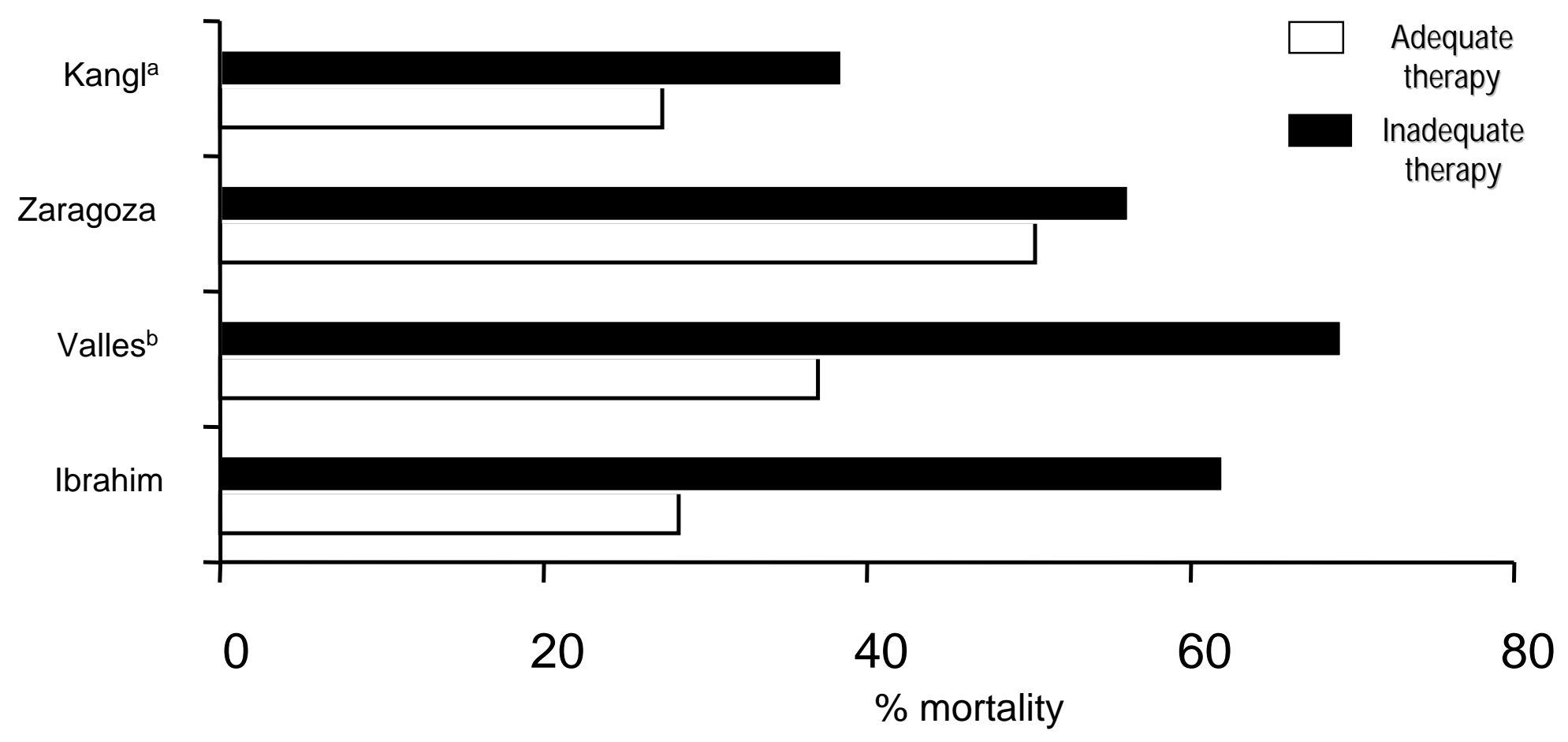

Kangl et al. Antimicrob Agents Chemother 2005;49:760-6 Zaragoza et al. Clin Microbiol Infect 2003;9:412-18 Valles et al. Chest 2003;123:1615-24 Ibrahim et al. Chest 2000;118:146-55 